\title{
Random Multi-Overlap Structures and Cavity Fields in Diluted Spin Glasses
}

\author{
Luca De Sanctis *
}

October 3, 2018

\begin{abstract}
We introduce the concept of Random Multi-Overlap Structure (RaMOSt) as a generalization of the one introduced by M. Aizenman R. Sims and S. L. Starr for non-diluted spin glasses. We use such method to find generalized bounds for the free energy of the Viana-Bray model of diluted spin glasses and to formulate and prove the Extended Variational Principle that implicitly provides the free energy of the model. Then we exhibit a theorem for the limiting RaMOSt, analogous to the one found by F. Guerra for the Sherrington-Kirkpatrick model, that describes some stability properties of the model. Last, we show how our technique can be used to prove the existence of thermodynamic limit of the free energy. The present work paves the way to a revisited Parisi theory for diluted spin systems.
\end{abstract}

Key words and phrases: diluted spin glasses, overlap structures, cavity fields, generalized bound, extended variational principle.

\section{Introdution}

The diluted mean field spin glasses are important both for their correspondence to random optimization problems and for their sort of intermediate nature halfway from idealized mean field models to short range realistic ones, thanks to the finite degree of connectivity.

Among the few rigorous results obtained so far in diluted spin glasses, two important examples are ref. [3, 5], where S. Franz and M. Leone found bounds for the free energy of diluted spin systems, considering the first level of Replica Symmetry Breaking; while D. Panchenko and M. Talagrand

\footnotetext{
*Department of Mathematics, Princeton University, Fine Hall, Washington Road, Princeton NJ 08544-1000 USA <lde@math.princeton.edu>
} 
found a way to consider any level Broken Replica Symmetry Bound in a compact way, using a weighting scheme inspired by ref. [1. In the high temperature region, rigorous results have been obtained for the K-Sat model of diluted spin glass by M. Talagrand 7 and by F. Guerra and F. L. Toninelli for the Viana-Bray model 4 .

In the case of non-diluted models, M. Aizenman, R. Sims and S. L. Starr recenlty introduced the concept of ROSt (Random Overlap Structures) through which they found bounds for the free energy in a very elegant and easy manner. In the same important paper ${ }^{1}$, the authors expressed the solution through an Extended Variational Principle. An important restriction of the ROSt space has been done by F. Guerra 2], exhibiting invariance of the limiting ROSt under certain transformations.

After the introduction of the basic definitions in section [2 we extend the ideas of ref. 1] to diluted spin glasses, in section 3 The finite connectivity requires that we consider Multi-Overlap as opposed to Overlap Structures (because the couplings are not Gaussian). In section 4 we prove a generalized bound for the free energy of the Viana-Bray model, by means of an interpolation not based on the iterative approach of ref. [6] used to find bounds in ref. 3, 5. Rather, our interpolation is closer to the one used for non-diluted models in ref. [1. As a consequence we can (like in ref. [1]) formulate an Extended Variational Principle for the free energy. The next natural step we performed, in section [5 is the search for invariant transformations of the optimal limiting RaMOSt, and we found stability properties similar to those found for non-diluted systems in ref. [2]. Appendix $\mathrm{A}$ is devoted to a calculation that plays a basic role throughout the paper, Appendix B contains a somewhat new proof of the existence of the thermodynamical limit of the free energy, Appendix $[$ reports some comments about optimal versus non-optimal RaMOSt's in terms of the phenomenon of overlap coalescence and generalized trial functions.

\section{Model, Notations, Definitions}

We refer to ref. 44 for an introduction to the Viana-Bray model, a physical description, the role of replicas and multi-overlaps, the infinite connectivity limit and the connection to the Sherrington-Kirkpatrick model, the behavior in the annealed region.

We will have in mind a lattice with a large bulk of $N$ sites (cavity) and $M$ additional spins ( $N$ is large and $M$ is fixed).

Notations:

$\alpha, \beta, h$ are non-negative real numbers (degree of connectivity, inverse temperature and external field respectively);

$P_{\zeta}$ is a Poisson random variable of mean $\zeta$; 
$\left\{i_{\nu}\right\},\left\{l_{\nu}\right\}$ are independent identically distributed random variables, uniformly distributed over the cavity points $\{1, \ldots, N\}$;

$\left\{j_{\nu}\right\},\left\{k_{\nu}\right\}$ are independent identically distributed random variables, uniformly ditributed over the added points $\{1, \ldots, M\}$;

$\left\{J_{\nu}\right\},\left\{\hat{J}_{\nu}\right\},\left\{\tilde{J}_{\nu}\right\}, J$ are independent identically distributed random variables, with symmetric distribution;

$\mathcal{J}$ is the set of all the quenched random variables above;

$\sigma: j \rightarrow \sigma_{j}= \pm 1, \tau: i \rightarrow \tau_{i}= \pm 1$ are the added and cavity spin configurations respectively; $\rho$. will be used for the spins in the full lattice (the points of which are denoted by $r, s$ ) without distinguishing between cavity and added spins;

$\pi_{\zeta}(\cdot)$ is the Poisson measure of mean $\zeta$.

$\mathbb{E}$ is an average over all (or some of) the quenched variables;

$\omega_{\mathcal{J}}$ is the Bolztmann-Gibbs average explicitly written below;

$\Omega_{K}$ is a product of the needed number of independent identical copies (replicas) of $\omega_{\mathcal{J}}$, in a system with $K$ spins;

$\langle\cdot\rangle$ will indicate the composition of an $\mathbb{E}$-type average over some quenched variables and some sort of Boltzmann-Gibbs average over the spin variables, to be specified each time.

We will often drop the dependance on some variables or indices or slightly change notations to lighten the expressions, when there is no ambiguity.

In absence of external field, the Hamiltonian of the system of $M$ sites is, by definition

$$
H_{M}(\sigma, \alpha ; \mathcal{J})=-\sum_{\nu=1}^{P_{\alpha M}} J_{\nu} \sigma_{j_{\nu}} \sigma_{k_{\nu}}
$$

When there is an external field $h$, the Hamiltonian is $H_{M}+H_{M}^{e x t}$, where we used the definition $H_{M}^{e x t}(\sigma, h)=-h \sum_{j=1}^{M} \sigma_{j}$.

We follow the usual basic definitions and notations of thermodynamics for the partition function and the free energy per site

$$
\begin{aligned}
Z_{M}(\beta, \alpha, h ; \mathcal{J}) & =\sum_{\{\sigma\}} \exp \left(-\beta\left(H_{M}(\sigma, \alpha ; \mathcal{J})+H_{M}^{e x t}(\sigma, h)\right)\right), \\
-\beta f_{M}(\beta, \alpha, h) & =\frac{1}{M} \mathbb{E} \ln Z_{M}(\beta, \alpha ; \mathcal{J})
\end{aligned}
$$

and $f=\lim _{M} f_{M}$.

The Boltzmann-Gibbs average of an observable $\mathcal{O}$ is

$$
\omega_{\mathcal{J}}(\mathcal{O})=Z_{M}(\beta, \alpha, h ; \mathcal{J})^{-1} \sum_{\{\sigma\}} \mathcal{O}(\sigma) \exp \left(-\beta\left(H_{M}(\sigma, \alpha ; \mathcal{J})+H_{M}^{e x t}(\sigma, h)\right)\right)
$$


The multi-overlaps are defined (using replicas) by

$$
q_{n}=\frac{1}{N} \sum_{i=1}^{N} \tau_{i}^{(1)} \cdots \tau_{i}^{(n)}, \tilde{q}_{n}=\frac{1}{M} \sum_{j=1}^{M} \sigma_{j}^{(1)} \cdots \sigma_{j}^{(n)}
$$

We are going to use the two following independent auxiliary Hamiltonians:

$$
\begin{aligned}
& \kappa(\tau, \alpha ; \mathcal{J})=-\sum_{\nu=1}^{P_{\alpha M}} \hat{J}_{\nu} \tau_{i_{\nu}} \tau_{l_{\nu}} \\
& \eta(\tau, \sigma, \alpha ; \mathcal{J})=-\sum_{\nu=1}^{P_{2 \alpha M}} \tilde{J}_{\nu} \tau_{i_{\nu}} \sigma_{j_{\nu}} \equiv \sum_{j=1}^{M} \eta_{j} \sigma_{j}
\end{aligned}
$$

where $\eta_{j}$ is the Cavity Field acting on $\sigma_{j}$ defined by

$$
\eta_{j}=\sum_{\nu=1}^{P_{2 \alpha}} J_{\nu}^{j} \tau_{i_{\nu}^{j}}
$$

and the index $j$ of $J_{\nu}^{j}$ and $\tau_{i_{\nu}^{j}}$ de-numerates independent copies of the corresponding random variables.

The two expressions of $\eta$ define the same random variable, but the first is probably the most convenient for the calculations in next two sections, while the second describes better the physics of the model, and will be essential in section 5 .

\section{Generalized Bound for the Free Energy}

For $t \in[0,1]$, consider the following Interpolating Hamiltionian

$$
H(t)=H_{M}(t \alpha)+\kappa(t \alpha)+\eta((1-t) \alpha)+H_{M}^{e x t}
$$

and using a set of weights $\xi_{\tau}$ define

$$
R_{M}(t)=\frac{1}{M} \mathbb{E} \ln \frac{\sum_{\tau, \sigma} \xi_{\tau} \exp (-\beta H(t))}{\sum_{\tau} \xi_{\tau} \exp (-\beta \kappa)}
$$

Call $G_{M}$ the value of $R_{M}$ at $t=0$

$$
G_{M}(\beta, \alpha, h ; \xi)=\frac{1}{M} \mathbb{E} \ln \frac{\sum_{\tau, \sigma} \xi_{\tau} \exp \left(-\beta\left(\eta+H_{M}^{e x t}\right)\right)}{\sum_{\tau} \xi_{\tau} \exp (-\beta \kappa)}
$$

then

$$
\begin{aligned}
& R_{M}(0)=G_{M} \\
& R_{M}(1)=-\beta f_{M}
\end{aligned}
$$




\section{Theorem 1 (Generalized Bound)}

$$
-\beta f \leq \lim _{M \rightarrow \infty} \inf _{\xi} G_{M}
$$

\section{Proof}

The proof is based on Lemma 1 Appendix $\mathrm{A}$

Define

$$
\Upsilon\left(m_{1}, m_{2}, m_{3}\right)=\exp \left(\beta\left(\sum_{\nu=1}^{m_{1}} J_{\nu} \sigma_{j_{\nu}} \sigma_{k_{\nu}}+\sum_{\nu=1}^{m_{2}} \hat{J}_{\nu} \tau_{i_{\nu}} \tau_{l_{\nu}}+\sum_{\nu=1}^{m_{3}} \tilde{J}_{\nu} \tau_{i_{\nu}} \sigma_{j_{\nu}}\right)\right)
$$

Let us compute the $t$-derivative of $R_{M}$, keeping in mind that its denominator does not depend on $t$.

$$
\begin{aligned}
& \frac{d}{d t} R_{M}(t)=\frac{d}{d t} \frac{1}{M} \mathbb{E} \ln \frac{\sum_{\tau, \sigma} \xi_{\tau} \Upsilon\left(P_{t \alpha M}, P_{t \alpha M}, P_{(1-t) 2 \alpha M}\right)}{\sum_{\tau} \xi_{\tau} \exp (-\beta \kappa)}= \\
& \frac{1}{M} \sum_{\{m .\}}^{0, \infty} \frac{d}{d t} \pi_{t \alpha M}\left(m_{1}\right) \pi_{t \alpha M}\left(m_{2}\right) \pi_{(1-t) 2 \alpha M}\left(m_{3}\right) \mathbb{E} \ln \sum_{\tau, \sigma} \xi_{\tau} \Upsilon\left(m_{1}, m_{2}, m_{3}\right)
\end{aligned}
$$

Now we have the sum of three terms, in each of which one of the $\pi$ 's is differentiated with respect to $t$. As in Appendix $\$$ we can substitute into the first term the following relation

$$
\Upsilon\left(m_{1}, m_{2}, m_{3}\right)=\exp \left(\beta J_{m_{1}} \sigma_{j_{m_{1}}} \sigma_{k_{m_{1}}}\right) \Upsilon\left(m_{1}-1, m_{2}, m_{3}\right)
$$

and we can do the same for the other two terms.

It is clear then that as in Lemma 1 of Appendix $\mathrm{A}$ we get an average $\Omega_{\xi, t}$ with weights consisting of the weights $\xi_{\tau}$ times the Boltzmann-Gibbs weights associated to $H(t)$ :

$$
\begin{aligned}
& \frac{d}{d t} R_{M}(t)= \\
& \alpha\left[\mathbb{E} \ln \Omega_{\xi, t} \exp \left(\beta J \sigma_{j} \sigma_{k .}\right)+\mathbb{E} \ln \Omega_{\xi, t} \exp \left(\beta J \tau_{i,} \tau_{l .}\right)-2 \mathbb{E} \ln \Omega_{\xi, t} \exp \left(\beta J \tau_{i,} \sigma_{j .}\right)\right]
\end{aligned}
$$

According to Appendix $\mathrm{A}$ we will get now some terms in $\cosh (\beta J)$, such terms can be factorized out, but here they are cancelled since they sum up to zero. Since

$$
\mathbb{E} \omega_{t}^{2 n}\left(\sigma_{j} \sigma_{k}\right)=\left\langle\tilde{q}_{2 n}^{2}\right\rangle_{t}, \mathbb{E} \omega_{t}^{2 n}\left(\tau_{i} \tau_{l .}\right)=\left\langle q_{2 n}^{2}\right\rangle_{t}, \mathbb{E} \omega_{t}^{2 n}\left(\tau_{i} \sigma_{j}\right)=\left\langle q_{2 n} \tilde{q}_{2 n}\right\rangle_{t}
$$

following the last steps of Lemma 1 we finally get

$$
\frac{d}{d t} R_{M}(t)=-\alpha \sum_{n=1}^{\infty} \frac{1}{2 n} \mathbb{E} \tanh ^{2 n}(\beta J)\left\langle\left(q_{2 n}-\tilde{q}_{2 n}\right)^{2}\right\rangle_{t}
$$


Thus

$$
\frac{d}{d t} R_{M}(t) \leq 0
$$

which implies $R_{M}(1) \leq R_{M}(0)$, i.e.

$$
-\beta f_{M} \leq G_{M}
$$

for all $\xi$ and $M$, hence

$$
-\beta f_{M} \leq \inf _{\xi} G_{M}
$$

Notice that we could obtain the same bounds using any other $\eta$ and $\kappa$ leading to the bound (6). Even more is true, we could pre-assign the values $q_{n}$ and forget that they are overlaps of configurations in a lattice with $N$ spins, which therefore is not an essential setting. Such remark explains the introduction of the following 1

Definition $1 A$ Random Multi-Overlap Structure $\mathcal{R}$ is a triple $\left(\Sigma,\left\{q_{n}\right\}, \xi\right)$ where

- $\Sigma$ is a discrete space;

- $\xi: \Sigma \rightarrow \mathbb{R}_{+}$is a system of random weights;

- $q_{n}: \Sigma^{n} \rightarrow[0,1], n \in \mathbb{N},|q| \leq 1$ is a positive definite Multi-Overlap Kernel (equal to 1 only on the diagonal of $\Sigma^{n}$ ).

Sometimes one considers the closure of $\Sigma$, which is not discrete in general. For any RaMOSt one takes a couple of auxiliary random variables compatible with the Multi-Overlap Kernel and with ([6]), and the previous theorem could be stated ${ }^{1}$ as

$$
-\beta f_{M} \leq \inf _{\mathcal{R}} G_{M}
$$

The generality of the RaMOSt allows to take $\Sigma$ (which is not necessarily $\left.\{-1,1\}^{N}\right)$ as the set of indexes of the weights $\xi_{\gamma}, \gamma \in \Sigma$ constructed by means of Random Probability Cascades of Poisson-Dirichlet processes (see e.g. ref. [5]). A well known property of these Cascades (see e.g. equation 4.2 in ref. [5]) gives place to a chain of expectations of Parisi type (see e.g. equation 5.5 in ref. [5]), that coincides with the Parisi Replica Symmetry Breaking theory if one interpolates according to the iterative approach of ref. 6, like in ref. 3, 5]. Since we interpolate with different auxiliary Hamiltonians, in our case (taking identical copies of $\eta^{\gamma}$ and $\kappa^{\gamma}$ in (3) and summing also over $\gamma$ ) the chain of expectations is not equivalent to the Parisi Replica Symmetry Breaking in the sense of [6]. This point will be deepened elsewhere $\frac{8}{}$. In order to get the Parisi Replica Symmetry 
Breaking theory in the traditional sense, one should take (like in ref. [1] for non-diluted models) a sequence of suitably chosen $\eta^{\gamma}$ and $\kappa^{\gamma}$ such that the corresponding bounds would be the special realization of (17) with the Parisi RaMOSt, like in ref. [1] for non-diluted models.

\section{Extended Variational Principle}

We can express the free energy of the model in the form of the following

\section{Theorem 2 (Extended Variational Principle)}

$$
-\beta f=\lim _{M \rightarrow \infty} \inf _{\mathcal{R}} G_{M}
$$

In order to prove the Extended Variational Principle, we will find, in Theorem 3 below, the opposite bound to (7), like in ref. [1].

Notice first that the following Cesàro limit can be easily computed

$$
\mathbf{C} \lim _{N} \frac{1}{M} \mathbb{E} \ln \frac{Z_{N+M}}{Z_{N}}=-\beta f
$$

since, thanks to the cancellation of the common terms of the numerator and denominator

$$
\begin{aligned}
\frac{1}{N} \sum_{K=1}^{N} & \frac{1}{M} \mathbb{E} \ln \frac{Z_{K+M}}{Z_{K}}= \\
\frac{1}{N} & \frac{1}{M}\left(\mathbb{E} \ln Z_{N+M}+\cdots+\mathbb{E} \ln Z_{N+1}-\mathbb{E} \ln Z_{1}-\cdots-\mathbb{E} \ln Z_{M}\right)
\end{aligned}
$$

and the first $M$ terms, with the positive sign, tend to $-\beta f$; while the others, with the negative sign, vanish in the limit.

Theorem 2 will be proven if we prove the following

\section{Theorem 3 (Reversed Bound)}

$$
-\beta f \geq \lim _{M \rightarrow \infty} \inf _{\mathcal{R}} G_{M}
$$

\section{Proof}

If we prove the statement for the restricted RaMOSt space of the $\mathcal{R}$ 's such that $\xi_{\tau}$ is 1 the Boltzmann-Gibbs factor

$$
\xi_{\tau}=\exp \left[-\beta\left(H_{N}(\tau)+H_{N}^{e x t}(\tau)\right)\right] \equiv \bar{\xi}_{N}
$$

then the theorem will hold a fortiori. Hence, given (8), it is enough to show

$$
\mathbf{C} \lim _{N} \frac{1}{M} \mathbb{E} \ln \frac{Z_{N+M}}{Z_{N}} \geq\left.\liminf _{N} G_{M}\right|_{\xi_{\tau}=\bar{\xi}_{N}}
$$


We can re-write $G_{M}$ as

$$
\frac{1}{M} \mathbb{E} \ln \left[\frac{\sum_{\tau, \sigma} \exp \left(-\beta\left(H_{N}+\eta\right)\right)}{Z_{N+M}\left(\alpha^{\prime}\right)} \frac{Z_{N+M}\left(\alpha^{\prime}\right)}{Z_{N+M}(\alpha)} \frac{Z_{N+M}(\alpha)}{Z_{N}(\alpha)} \frac{Z_{N}(\alpha)}{\sum_{\tau} \exp \left(-\beta\left(H_{N}+\kappa\right)\right)}\right]
$$

therefore we have four terms.

The rest of the proof is very similar to the proof of Theorem 4 in the next section.

Recall that the sum of Poisson random variables is a Poisson random variable with mean equal to the sum of the means. So if we take

$$
\alpha^{\prime}=\alpha \frac{(N+M)}{N}
$$

we see that the forth fraction is the same as $Z_{N}(\alpha) / Z_{N}\left(\alpha^{\prime}\right)$. Furthermore, since $\alpha^{\prime}(N+M)-\alpha(N+M)=\alpha^{\prime} N-\alpha N, \alpha^{\prime} \rightarrow \alpha$, in the limit the second and forth fractions cancel out thanks to Lemma 1 in Appendix $\mathrm{A}$

We have seen that the third fraction tends to $-\beta f$.

Now we proceed with a key step of our approach. In the denominator of the first fraction we can split the mean $\alpha^{\prime}(N+M)$ into the sum of three means such that $H_{N+M}$ splits into the sum of three Hamiltonians with the first depending only on cavity spins $\tau$, the second containing interactions between the cavity and the added spins, the third has the added spins only. In other words, we are considering the fraction of the interactions (indexed by $\nu$ ) within the cavity, between the cavity and added spins, within the added lattice respectively. Hence the new three means will be proportional to $N^{2}, 2 N M, M^{2}$ respectively. More explicitly

$$
\begin{aligned}
& Z_{N+M}\left(\alpha^{\prime}\right)=\sum_{\rho} \exp \left(\beta \sum_{\nu=1}^{P_{\alpha^{\prime}(N+M)}} J_{\nu} \rho_{r_{\nu}} \rho_{s_{\nu}}\right)= \\
& \sum_{\tau, \sigma} \exp \beta\left(\sum_{\nu=1}^{P_{\breve{\zeta}}} J_{\nu} \tau_{i_{\nu}} \tau_{l_{\nu}}+\sum_{\nu=1}^{P_{\tilde{\zeta}}} J_{\nu} \tau_{i_{\nu}} \sigma_{j_{\nu}}+\sum_{\nu=1}^{P_{\hat{\zeta}}} J_{\nu} \sigma_{j_{\nu}} \sigma_{k_{\nu}}\right)
\end{aligned}
$$

where

$$
\breve{\zeta}=\alpha^{\prime} \frac{N^{2}}{N+M}, \tilde{\zeta}=\alpha^{\prime} \frac{2 N M}{N+M}, \hat{\zeta}=\alpha^{\prime} \frac{M^{2}}{N+M}
$$

The third Hamiltonian is thus negligible. This means that when the cavity is large the added spins do not interact one another.

The choice of $\alpha^{\prime}$ we made guarantees that numerator and denominator contain two (up to a negligible third in the denominator) identical Hamiltonians with the same connectivities. As a consequence, the first fraction in $G_{M}$ vanishes in the limit and the theorem is proven. 


\section{Limiting RaMOSt Invariance}

As in Appendix $\AA$

$$
A=\mathbb{E} \ln \cosh (\beta J)-\sum_{n=1}^{\infty} \frac{1}{2 n} \mathbb{E} \tanh ^{2 n}(\beta J)\left\langle q_{2 n}^{2}\right\rangle
$$

Then we can state the following

Theorem 4 In the whole region where the parameters are uniquely defined, the following Cesàro limit is linear in $M$ and $\bar{\alpha}$

$$
\mathbf{C} \lim _{N} \mathbb{E} \ln \Omega_{N}\left\{\sum_{\sigma} \exp [-\beta(\eta(\alpha)+\kappa(\bar{\alpha}))]\right\}=M(-\beta f+\alpha A)+\bar{\alpha} A
$$

\section{Proof}

The proof is based on the comparison between the limit above and (9), similarly to the method of the previous section. More precisely, the left hand side of (12) can be re-written (without the limit) as

$$
\begin{aligned}
& \mathbb{E} \ln \Omega_{N} \Upsilon\left(0, P_{\alpha N+\bar{\alpha}}, P_{2 \alpha M}\right)= \\
& \mathbb{E} \ln \frac{\sum_{\tau, \sigma} \Upsilon\left(0, P_{\alpha N+\bar{\alpha}}, P_{2 \alpha M}\right)}{Z_{N+M}\left(\alpha^{\prime}\right)} \frac{Z_{N+M}\left(\alpha^{\prime}\right)}{Z_{N+M}(\alpha)} \frac{Z_{N+M}(\alpha)}{Z_{N}(\alpha)}= \\
& \mathbb{E} \ln \frac{\sum_{\tau, \sigma} \Upsilon\left(0, P_{\alpha N+\bar{\alpha}}, P_{2 \alpha M}\right)}{\sum_{\tau, \sigma} \Upsilon\left(P_{\alpha^{\prime} \frac{M^{2}}{(N+M)}}, P_{\alpha^{\prime} \frac{N^{2}}{(N+M)}}, P_{\alpha^{\prime}} \frac{2 N M}{(N+M)}\right)} \frac{Z_{N+M}\left(\alpha^{\prime}\right)}{Z_{N+M}(\alpha)} \frac{Z_{N+M}(\alpha)}{Z_{N}(\alpha)}
\end{aligned}
$$

We know that the third fraction will give $-\beta f M$. But is is also clear that if we take

$$
\alpha^{\prime}=\frac{(N+M)}{N^{2}}(\alpha N+\bar{\alpha})
$$

the three parameters in the numerator of the first fraction tend to the corresponding ones in the denominator, so that the first fraction is immaterial in the limit. Now notice that

$$
\alpha^{\prime}(N+M)-\alpha(N+M) \rightarrow \alpha M+\bar{\alpha}
$$

and therefore thanks to Lemma 1 of Appendix $\$$ the contribution of the second fraction is

$$
(\alpha M+\bar{\alpha}) A
$$

If we now write

$$
\sum_{\sigma} \exp (-\beta \eta)=\prod_{j=1}^{M} 2 \cosh \left(\beta \sum_{\nu=1}^{P_{2 \alpha}} J_{\nu}^{j} \tau_{i_{\nu}^{j}}\right) \equiv c_{1} \cdots c_{M}
$$


we can formulate (12) as

$$
\mathbf{C} \lim _{N} \mathbb{E} \ln \Omega_{N}\left\{c_{1} \cdots c_{M} \exp \kappa(\bar{\alpha})\right\}=M(-\beta f+\alpha A)+\bar{\alpha} A
$$

from which it is clear that each cavity field (more precisely each $c_{j}$ ) yields a contribution $(-\beta f+\alpha A)$ in the limit.

Notice that in the limiting structure not only the cavity fields are mutually independent, but they are independent of $\kappa$ as well. We have thus obtained the analogy with the result of F. Guerra regarding the SherringtonKirkpatrick model 2

\section{Conclusions}

It is important now to formulate in a complete manner the Parisi theory

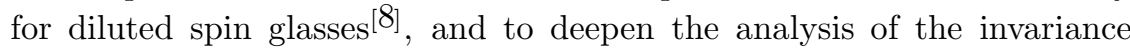
properties of the optimal RaMOSt in order to characterize the solution. We plan to dedicate future work to this program.

\section{A Connectivity Shift}

Lemma 1 Let $\alpha^{\prime} N=\alpha(N+\Lambda)$, with $\Lambda / N \rightarrow 0$ as $N \rightarrow \infty$. Then, in the whole region where the parameters are uniquely defined

$$
\lim _{N} \mathbb{E} \ln \frac{Z_{N}\left(\alpha^{\prime}\right)}{Z_{N}(\alpha)}=\Lambda\left[\mathbb{E} \ln \cosh (\beta J)-\sum_{n=1}^{\infty} \frac{\left\langle q_{2 n}^{2}\right\rangle}{2 n} \mathbb{E} \tanh ^{2 n}(\beta J)\right]
$$

\section{Proof}

The proof is based on standard convexity arguments.

For $t \in[0,1]$, define

$$
\alpha_{t}^{\prime}=\alpha\left(1+t \frac{\Lambda}{N}\right)
$$

so that $\alpha_{t}^{\prime} \rightarrow \alpha$ as $N \rightarrow \infty$.

We have

$$
A_{t} \equiv \mathbb{E} \ln \frac{Z_{N}\left(\alpha_{t}^{\prime}\right)}{Z_{N}(\alpha)}=\mathbb{E} \ln \Omega \exp (-\beta \kappa(\alpha t \Lambda))
$$

Let us compute the $t$-derivative of $A(t)$

$$
\frac{d}{d t} A_{t}=\mathbb{E} \sum_{m=0}^{\infty} \frac{d}{d t} \pi_{\alpha t \Lambda}(m) \ln \sum_{\tau} \exp \left(\beta \sum_{\nu=0}^{m} J_{\nu} \tau_{i_{\nu}} \tau_{l_{\nu}}\right)
$$

Using the following elementary property

$$
\frac{d}{d t} \pi_{t \zeta}(m)=\zeta\left(\pi_{t \zeta}(m-1)-\pi_{t \zeta}(m)\right)
$$


we get

$$
\begin{aligned}
\frac{d}{d t} A_{t} & =\alpha \Lambda \mathbb{E} \sum_{m=0}^{\infty}\left[\pi_{\alpha t \Lambda}(m-1)-\pi_{\alpha t \Lambda}(m)\right] \ln \sum_{\tau} \exp \left(\beta \sum_{\nu=1}^{m} J_{\nu} \tau_{i_{\nu}} \tau_{l_{\nu}}\right) \\
& =\alpha \Lambda \mathbb{E} \ln \sum_{\tau} \exp \left(\beta J \tau_{i_{\nu}} \tau_{l_{\nu}}\right) \exp \left(\beta \sum_{\nu=1}^{P_{\alpha t \Lambda}} J_{\nu} \tau_{i_{\nu}} \tau_{l_{\nu}}\right) \\
& -\alpha \Lambda \mathbb{E} \ln \sum_{\tau} \exp \left(\beta \sum_{\nu=1}^{P_{\alpha t \Lambda}} J_{\nu} \tau_{i_{\nu}} \tau_{l_{\nu}}\right) \\
& =\alpha \Lambda \mathbb{E} \ln \Omega_{t} \exp \left(\beta J \tau_{i_{\nu}} \tau_{l_{\nu}}\right)
\end{aligned}
$$

where we included the $t$-dependent weights in the average $\Omega_{t}$. Now use the following identity

$$
\exp \left(\beta J \tau_{i} \tau_{l}\right)=\cosh (\beta J)+\tau_{i} \tau_{l} \sinh (\beta J)
$$

to get

$$
\frac{d}{d t} A_{t}=\alpha \Lambda \mathbb{E} \ln \Omega_{t}\left[\cosh (\beta J)\left(1+\tanh (\beta J) \tau_{i_{\nu}} \tau_{l_{\nu}}\right)\right]
$$

We have already observed that

$$
\mathbb{E} \omega_{t}^{2 n}\left(\tau_{i,} \tau_{l .}\right)=\left\langle q_{2 n}^{2}\right\rangle_{t}
$$

so if we now expand the logarithm in power series, we see that the result does not depend on $t$ in the limit of large $N$, because $\alpha_{t}^{\prime} \rightarrow \alpha$ and hence $\Omega_{t} \rightarrow \Omega$. Therefore integrating back against $t$ from 0 to 1 is the same as multiplying by 1 . Thanks to the symmetric distribution of $J$ we get the result, where the odd powers are missing.

\section{B Thermodynamic Limit}

Our new interpolation method should allow one to prove the existence of the thermodynamic limit (already proven in ref. 3]), for otherwise it would be quite weak. As a matter of fact, this theorem turns out to be elementary with our interpolation.

It is well known that a sufficient condition for the existence of the thermodynamic limit is the sub-additivity of the free energy. In our context we want to measure the changes caused by adding the $M$ additional spins to the cavity of size $N$ and make sure that

$$
\mathbb{E} \ln Z_{N+M} \geq \mathbb{E} \ln Z_{N}+\mathbb{E} \ln Z_{M}
$$


The natural start is therefore considering the following interpolation

$$
\mathbb{E} \ln \sum_{s} \exp \left[-\beta\left(H_{N+M}(t \alpha)+H_{N}((1-t) \alpha)+H_{M}((1-t) \alpha)\right)\right] \equiv \Phi_{t}
$$

We clearly proceed by splitting $H_{N+M}$ in the usual way, and we get this time a total of five Hamiltonians. In the $t$-derivative the parts in $\cosh (\beta J)$ cancel out, since the sum of the five coefficients of $\alpha$ is zero. The remaining terms are

$$
\frac{d}{d t} \Phi_{t}=-\alpha \sum_{n=1}^{\infty} \frac{\mathbb{E} \tanh ^{2 n}(\beta J)}{2 n} B_{n, t}
$$

where

$B_{n, t}=\left\{\frac{1}{N+M}\left[\left\langle\left(q_{2 n} N\right)^{2}+2 q_{2 n} N \tilde{q}_{2 n} M+\left(\tilde{q}_{2 n} M\right)^{2}\right\rangle_{t}\right]-\left\langle q_{2 n}^{2} N+\tilde{q}_{2 n}^{2} M\right\rangle_{t}\right\}$

Now it is trivial to show that

$$
B_{n, t}=-\frac{1}{N+M} N M\left\langle\left(q_{2 n}-\tilde{q}_{2 n}\right)^{2}\right\rangle_{t} \leq 0
$$

\section{Sum Rules and Trial Functions}

Consider the function $\Phi_{t}$ defined in the previous section, then the fundamental theorem of calculus implies

$$
\mathbb{E} \ln Z_{N+M}-\mathbb{E} \ln Z_{N}=\mathbb{E} \ln Z_{M}+\int_{0}^{1} \frac{d \Phi_{t}}{d t} d t
$$

Dividing by $M$ and taking the Cesàro limit as $N$ goes to infinity yields the following sum rule

$$
-\beta f(\beta, \alpha)=-\beta f_{M}(\beta, \alpha)-\alpha \sum_{n=1}^{\infty} \frac{\mathbb{E} \tanh ^{2 n}(\beta J)}{2 n} \mathbf{C} \lim _{N} \int_{0}^{1}\left\langle\left(q_{2 n}-\tilde{q}_{2 n}\right)^{2}\right\rangle_{t} d t
$$

which shows that the difference $f-f_{M}$ is given in terms of the multi-overlap distance averaged over any optimal RaMOSt.

If we instead use the fundamental theorem of calculus for $R_{M}(t)$ defined in section 3 , we get another sum rule

$$
-\beta f_{M}=G_{M}(\mathcal{R}, \eta, \kappa)-\alpha \sum_{n=1}^{\infty} \frac{\mathbb{E} \tanh ^{2 n}(\beta J)}{2 n} \mathbf{C} \lim _{N} \int_{0}^{1}\left\langle\left(q_{2 n}-\tilde{q}_{2 n}\right)^{2}\right\rangle_{t} d t
$$

which explains, when $M$ goes to infinity, the role of $G_{M}(\mathcal{R}, \eta, \kappa)$ as trial function; minimizing it means finding optimal RaMOSt's (and therefore the free energy) and the multi-overlap locking (coalescence) described by Aizenman in the case of non-diluted models $\frac{9}{}$. 


\section{Acknoledgments}

The author warmly thanks Francesco Guerra for a priceless scientific exchange, and would also like to thank Fabio Lucio Toninelli for useful discussions, Edward Nelson and Yakov Sinai for support and availability, Michael Aizenman for encouragement and useful conversations, the Department of Physics at University of Rome "La Sapienza" (and in particular Giovanni Jona-Lasinio) for hospitality.

\section{References}

[1] M. Aizenman, R. Sims, S. L. Starr, An Extended Variational Principle for the SK Spin-Glass Model, Phys. Rev. B, 68, 214403 (2003)

[2] F. Guerra, About the Cavity Fields in Mean Field Spin Glass Models, ArXiv: cond-mat/0307673

[3] S. Franz, M. Leone, Replica bounds for optimization problems and diluted spin systems, J. Stat. Phys. 111535 (2003)

[4] F. Guerra, F. L. Toninelli, The high temperature region of the VianaBray diluted spin glass model, J. Stat. Phys. 115 (2004)

[5] D. Panchenko, M. Talagrand, Bounds for diluted mean-field spin glass models, to appear in Prob. Theory Related Fields

[6] M. Mezard, G. Parisi, The Bethe lattice spin glass revisited, Eur. Phys. J. B 20, 217 (2001)

[7] M.Talagrand, The high temperature case for the random K-sat problem, Prob. Theory Related Fields, 119:2, 187-212

[8] L. De Sanctis, F. Guerra, in preparation

[9] M. Aizenman, to appear in the proceedings volume of the conference "Equilibrium and Dynamics of Spin Glasses", Ascona, Switzerland. 